\title{
Generalization of the Activated Complex Theory of Reaction Rates. I. Quantum Mechanical Treatment
}

\author{
R. A. MARCUS* \\ Departments of Chemistry, Polytechnic Institute of Brooklyn, Brooklyn, New York, and Brookhaven National Laboratory, Upton, Nene York
}

(Received 18 June 1964)

\begin{abstract}
In its usual form activated complex theory assumes a quasiequilibrium between reactants and activated complex, a separable reaction coordinate, a Cartesian reaction coordinate, and an absence of interaction of rotation with internal motion in the complex. In the present paper a rate expression is derived without introducing the Cartesian assumption. The expression bears a formal resemblance to the usual one and reduces to it when the added assumptions of the latter are introduced. The new equation for the transmission coefficient contains internal centrifugal terms. The fourth assumption can also be weakened and a rotational interaction included in the formalism. In applications of the rate equation use can be made of the recent finding that in the immediate vicinity of a saddle point or a minimum, a potential energy surface can be imitated in some major topographical respects by a surface permitting separation of variables. The separated wave equation for the reaction coordinate is then curvilinear because of the usual curvature of the path of steepest ascent to the saddle point. Calculations of transmission coefficients and rates can be made and compared with those obtainable from the usual one-dimensional Cartesian-like calculations on the one hand and with some based on the numerical integration of the $n$-dimensional Schrödinger equation on the other. An application to a common three-center problem is discussed.
\end{abstract}

\section{INTRODUCTION}

$\mathbf{I}$ $\mathrm{N}$ activated complex theory the reaction coordinate has been assumed to be Cartesian and, in quantummechanical treatments at least, to be dynamically separable from the other coordinates. ${ }^{1,2}$ The effect of any rotational constants of the motion on the internal motion of the activated complex has normally been neglected, ${ }^{3}$

* This research was supported in part by a fellowship from the Alfred P. Sloan Foundation and by a grant from the U.S. Atomic Energy Commission. A portion of this research was performed under the auspices of the U.S. Atomic Energy Commission, while the author was a Visiting Senior Scientist at Brookhaven National Laboratory. New address: Noyes Chemical Laboratory, University of Illinois, Urbana, Illinois.

${ }^{1} \mathrm{H}$. Eyring, J. Chem. Phys. 3, 107 (1935); E. Wigner, Trans. Faraday Soc. 33, 29 (1937); E. Wigner, Z. Physik. Chem. B19, 203 (1932). M. G. Evans and M. Polanyi, Trans. Faraday Soc. 31, 875 (1935), employ an argument based on the partition function for the imaginary frequency, a partition function normally derived for a rectilinear (i.e., Cartesian) vibrational coordinate. G. H. Vineyard [J. Phys. Chem. Solids 3, 121 (1957)], gives an elegant classical formulation for site-to-site motion in crystals. He assumed the reaction coordinate to be rectilinear.

${ }^{2}$ In a classically based quasi-equilibrium treatment one considers dynamical properties of the system infinitesimally close to the $(n-1)$ dimensional hypersurface in configuration space defining the properties of an activated complex. (The total number of coordinates in configuration space is $n$.) In the quantum treatment, the implications of the uncertainty principle eliminate such considerations. One must employ instead a treatment based on the properties of the potential energy function over noninfinitesimal distances from the above hypersurface. In this case, however, the problem of separability of the equation of motion arises.

${ }^{3}$ For example, the angular momentum of the complex gives rise to a centrifugal potential whose influence on the vibrational motion is usually, and justifiably, ignored. There are some instances, such as in bimolecular reactions of negligible activation energy, where it should be and has been included in literature calculations, as well as in those on the reverse unimolecular dissociation. and equilibrium between reactants and activated complexes has been assumed. ${ }^{4}$

In the present paper and in a companion one ${ }^{5}$ on the classical-mechanical formulation, this activated complex theory is generalized by extending it to curvilinear reaction coordinates and, within certain limitations, ${ }^{6}$ by including the effect of the constants of the motion on the internal motion of the activated complex. The assumption of separability is made in the quantum formulation. It now has somewhat wider applicability than before, partly because of the availability of a local approximation method for nonseparable surfaces. ${ }^{7}$ Separability is not assumed in the classical-mechanical formulation, however. ${ }^{5}$

The desirability of extending activated complex theory to include curvilinear reaction coordinates is clear from an examination of the local topography of the potential energy surface near the saddle point, when that saddle point occurs: The path of steepest ascent to this point, the "reaction path," is almost in-

\footnotetext{
${ }^{4}$ In some problems, as in the unimolecular reaction of vibrationally excited molecules, a local equilibrium between a hot molecule and the activated complex for its reaction is assumed instead. [For example, R. A. Marcus and O. K. Rice, J. Phys. Colloid Chem. 55, 894 (1951); R. A. Marcus, J. Chem. Phys. 20, 359 (1952).]

${ }^{5}$ R. A. Marcus (to be published).

- The vibrational angular momentum is neglected. (The internal coordinates are chosen to satisfy the Eckart conditions, however.) See Footnote 15, Ref. 7.

${ }^{7}$ R. A. Marcus, J. Chem. Phys. 41, 610 (1964). The features which are matched are the tangent, the first curvature vector, the first curvature, and the force constant along each extremal path of ascent or descent to the saddle-point. Currently, the writer is extending these results by employing approximate separability over a larger region of coordinate space, to supplement the "almost exact" separability over the smaller region described in this reference.
} 
variably a curve in mass-weighted configuration space, rather than a straight line. In some major topographical respects this surface can be matched locally by one permitting separation of variables. ${ }^{7}$ The reaction coordinate is then found to be curvilinear. This coordinate is this approximately separable one near the saddle point, a coordinate which leads from the "reactants' region" of mass-weighted configuration space to the products' region in that neighborhood.

Except in computer calculations ${ }^{8}$ or in early discussions based on the motion of balls on surfaces, ${ }^{9}$ the dynamical effects of the curvature of the reaction path have been ignored in the literature. The curvilinear character gives rise dynamically to a centrifugal effect, an effect smaller at the saddle point than at a short distance from it, where the kinetic energy is greater. As discussed in detail elsewhere ${ }^{7}$ the net result is to make the reaction coordinate in mass-weighted configuration space have a smaller curvature than the reaction path. The centrifugal effect introduces a coupling between the reaction coordinate and the vibrations of the complex, just as the rotation of a molecule as a whole can influence the latter's vibration by a centrifugal potential. The above effect occurs in classical mechanics and, phrased in terms of probabilities, in quantum mechanics when the system has enough energy to surmount the barrier. At low energies nuclear tunneling occurs and, the formulas suggest, so does a nonclassical centrifugal effect, negative in nature.

We consider first the case where the dependence of the properties of the activated complex on the rotational constants of the motion can be neglected. In a later section the effect of the rotational state on the behavior of the activated complex is considered.

The basic equation, Eq. (21) below, reduces to the usual activated complex expression when the reaction coordinate is treated as a Cartesian one. Equation (21) may be used to consider several nonseparable $n$-dimensional problems by introducing into it the local approximation method mentioned earlier. ${ }^{10}$ Comparison with computer calculations then permit an assessment of the useful range of that local approximation and should also facilitate the physical interpretation of such calculations.

\section{SCHRODINGER EQUATION AND SEPARATED EQUATIONS}

Certain curvilinear coordinate systems will serve as better starting points for finding separable approximations to the nonseparable potential-energy function.

\footnotetext{
${ }^{8}$ Quantum calculations: E. M. Mortensen and K. S. Pitzer, Chem. Soc. (London) Spec. Publ. 16, 57 (1962). Classical calculations: F. T. Wall, L. A. Hiller, Jr., and J. Mazur, J. Chem. Phys. 29, 255 (1958), 35, 1284 (1961); N. C. Blais and D. L. Bunker, ibid. 39, 315 (1963) and references cited therein; $\mathbf{M}$. Karplus (to be published).

'S. Glasstone, K. J. Laidler, and H. Eyring, Theory of Rate Processes (McGraw-Hill Book Company, Inc., New York, 1940).

${ }^{10}$ Calculations are in progress by $\mathrm{J}$. Lane and R. A. Marcus for $n=2$.
}

We suppose that a coordinate in one of these has been selected as the reaction coordinate; a process of making the selection has been briefly discussed elsewhere. ${ }^{7}$ For example, in the case of a three-center-atomtransfer reaction involving a linear activated complex $(\mathrm{A}+\mathrm{BC} \rightarrow \mathrm{AB}+\mathrm{C})$ the reaction path in mass-weighted configuration space leading from reactants to products is a curved one. For such paths in the vicinity of the saddle-point region, circular cylinder, parabolic cylinder, elliptic cylinder, or other curvilinear coordinates are more appropriate than the usual Cartesian ones. Should both the vibrational path of steepest ascent from the critical point and the reaction path both be curved in this space, and should one wish to include this feature, elliptic cylinder coordinates would be more appropriate than circular cylinder ones. ${ }^{7}$

In curvilinear coordinates $q^{1}, \cdots, q^{n}$ the Schrödinger equation has the form ${ }^{11}$

$$
H \psi=\alpha_{1} \psi,
$$

where $H$ is the Hamiltonian operator, $\alpha_{1}$ the total energy, and $\psi$ the wavefunction of the entire system:

$$
H=\frac{-\hbar^{2}}{2} \sum_{s, t=1}^{n} \frac{1}{g^{\frac{1}{2}}} \frac{\partial}{\partial q^{s}} g^{\frac{1}{t}} g^{s t} \frac{\partial}{\partial q^{t}}+U .
$$

The $q^{s}$ are generalized coordinates, and $U$ is the potential energy; $g^{s t}$ is a contravariant tensor ${ }^{12}$ conjugate to the metric tensor $g_{s t}$ appearing in the line element $d s$ in mass-weighted space; $g$ is the determinant of the $g_{s t}$ :

$$
\begin{gathered}
g^{s t}=\sum_{i=1}^{n} \frac{1}{m^{i}} \frac{\partial q^{s}}{\partial x^{i}} \frac{\partial q^{t}}{\partial x^{i}} ; \quad g_{s t}=\sum_{i=1}^{n} m^{i} \frac{\partial x^{i}}{\partial q^{s}} \frac{\partial x^{i}}{\partial q^{t}}, \\
d s^{2}=\sum_{s, t=1}^{n} g_{s t} d q^{s} d q^{t} ; \quad \sum_{r=1}^{n} g_{s r} g^{r t}=\delta_{s}{ }^{t},
\end{gathered}
$$

where $x^{i}$ is a Cartesian coordinate of an atom of mass $m^{i}$; the coordinates of the $k$ th atom are given by $i=3 k$, $3 k+1,3 k+2$. Both $g_{s t}$ and $g^{s t}$ are symmetric tensors.

Under certain conditions on $U$ and on $g^{s t}$, Eq. (1) can be separated into $m$ individual equations, each depending on its own set of variables. ${ }^{13,138}$ The wavefunc-

\footnotetext{
${ }^{11}$ For example, W. Pauli, Jr., Handbuch der Physik, edited by S. Flügge (Springer-Verlag, Berlin, 1958), Vol. 5, p. 39. One sometimes sees Eq. (2) written instead with the right-hand side multiplied by $g^{\frac{1}{2}}$ on the left and by $g^{-\frac{1}{t}}$ on the right. The $\psi$ of the new equation exactly equals the original $\psi$ multiplied by $g^{t}$. Both equations have identical eigenvalues and transmission coefficients.

${ }^{12}$ For example, A. J. McConnell, Applications of Tensor Calculus (Dover Publications, Inc., New York, 1957).

${ }_{13}^{13}$ R. A. Marcus, J. Chem. Phys. 41, 603 (1964). Appendix II there shows how the formalism embodied in the equations of the present paper, (5) to (13), includes the influence of rotation in the case of a diatomic rotating-vibrating molecule.

${ }_{13 \mathrm{a}}$ Note added in proof: The present derivation of Eqs. (21) and (22) employs an extension ${ }^{13}$ of the Stäckel-Robertson formalism for separation of variables, embodied in Eqs. (5) to (13). The writer has since derived Eq. (22) without explicit introduction of the Stäckel-Robertson formalism but with retention of the basic assumption of an approximately separable reaction coordinate. This modified derivation will be summarized in Part III.
} 
tion $\psi$ then becomes

$$
\psi=\prod_{\mu=1}^{m} \psi_{\mu},
$$

where $\psi_{\mu}$ is the wavefunction for the $\mu$ th set. As a particular case of this separation one could select one set of variables to consist of a single variable, the reaction coordinate, $q^{r}$, and select a second set to consist of all remaining coordinates. That is, $m=2$ then. Under the assumed conditions ${ }^{13} g^{s t}$ vanishes when $s$ and $t$ belong to different sets.

As a result of the separation the Hamiltonian operator has the form (6)..$^{13}$

$$
H=\sum_{\mu=1}^{m}\left(\phi^{\mu 1} / f_{\mu}\right) H_{\mu}+U,
$$

where $H_{\mu}$ is a Hermitian differential operator (7). The potential energy $U$ is of the form (8), and $\phi^{\mu 1}$ and $f_{\mu}$ are defined later by Eqs. (11) and (13). ( $f_{\mu}$ is the factor in $g^{\frac{1}{2}} / \phi$ depending only on the coordinates belonging to the set $\mu$.)

$$
\begin{aligned}
H_{\mu} & =\frac{-\hbar^{2}}{2} \sum_{i, j=1}^{h_{\mu}} \frac{\partial}{\partial q^{\mu_{i}}} f^{\mu_{i} \mu_{j}} \frac{\partial}{\partial q^{\mu_{j}}}, \\
U & =\sum_{\mu=1}^{m} \phi^{\mu 1} X_{\mu} .
\end{aligned}
$$

The separated equations are

$$
\left(H_{\mu}+X_{\mu} f_{\mu}\right) \psi_{\mu}=\sum_{\nu=1}^{m} \alpha_{\nu} \phi_{\mu \nu} f_{\mu} \psi_{\mu} .
$$

$q^{\mu_{i}}$ is the $i$ th coordinate in Set $\mu$ (there are $h_{\mu}$ such coordinates), and $f^{\mu_{i} \mu_{j}}$ is defined by (12). The quantities $\phi_{\mu \nu}$ and $f^{\mu_{i} \mu_{j}}$ depend only on the properties of the metric. They are functions of the coordinates in the $\mu$ th set only and are independent of the potential-energy function; $X_{\mu}$ also depends only on the coordinates of Set $\mu$. The $\phi_{\mu \nu}$ are conjugate to the $\phi^{\mu \nu}$ and may be called the Stäckel coefficients ${ }^{14}$

$$
\begin{gathered}
\sum_{\mu=1}^{m} \phi^{\mu \nu} \phi_{\mu \lambda}=\delta_{\lambda}{ }^{\nu} . \\
\phi^{\mu 1}=\left(\operatorname{det} g^{\mu_{i} \mu_{j}}\right)^{1 / h_{\mu}},
\end{gathered}
$$

where $i$ and $j=1$ to $h_{\mu}$.

$$
f^{\mu_{i} \mu_{i}}=g^{\mu_{i} \mu_{j}} f_{\mu} /\left(\operatorname{det} g^{\mu_{i} \mu_{j}}\right)^{1 / h_{\mu}} .
$$

The determinant of the $\phi_{\mu \nu}, \phi$, is related to $g$ and to the $f_{\mu}$ :

$$
g^{\frac{1}{2}}=\phi \prod_{\mu=1}^{m} f_{\mu}
$$

${ }^{14}$ P. G. Stäckel, Habilitationsschrift, Halle, Germany (1891); Ann. Mat. Pure Appl. Ser. 2A 25, 55 (1897).
Various coordinate systems for which the StäckelRobertson separation of variables can be made have been described by Eisenhart ${ }^{15,16}$ for the case where $h_{\mu}=1$ for all $\mu$. He has given the corresponding $\phi_{\mu \nu}$ 's and $f_{\mu}$ 's and his results can be at least partly adapted to the present case where $h_{\mu}$ need not be unity. Typical approximations in the literature of molecular dynamics correspond, incidentally, to setting many of the $\phi_{\mu \nu}$ 's equal to zero and many of the $g^{r s}$ equal to zero or to constants.

\section{LOCAL APPROXIMATION}

As mentioned earlier it is supposed that it has been possible to describe a coordinate system such that one of the coordinates is approximately dynamically separable from all remaining coordinates in the activated complex region and tends to lead in this vicinity from the reactants' "region" to the products' one. This coordinate is then the "reaction coordinate." The potential energy surface is thereby approximated by one which permits separation of variables. ${ }^{17}$ The reaction coordinate forms one of the sets $\mu$ mentioned earlier. It is described by setting $\mu$ equal to $r$. Since only the nonseparable potential energy surface in the vicinity of the activated complex region is being approximated by a separable surface, the properties in the separated system for the degrees of freedom other than $q^{r}$ are those of the activated complex rather than of the reactants.

Parenthetically, it may be noted that this matching of the two energy surfaces will normally be poorer for configurations far from the activated complex region. However, if most of the scattering of the incident wave along the reaction coordinate occurs near the activated complex region, this poor fit for configurations remote from those of the complex is only of minor concern. Since the wavelength (more precisely, the reciprocal of the component of the wave vector along $q^{r}$ ) is large in the activated complex region because of the low kinetic energy there, and since the potential energy changes rapidly there, most of the scattering may in fact occurs in that region. Indeed, the phase integral expression ${ }^{18}$ for tunneling points up this local scattering characteristic.

${ }^{15}$ L. P. Eisenhart, Ann. Math. 35, 284 (1934).

${ }^{16}$ For example, P. M. Morse and H. Feshbach, Methods of Theoretical Physics (McGraw-Hill Book Company, Inc., New York, 1953), p. $655 \mathrm{ff}$.

${ }^{17}$ This procedure is a generalization of the customary one of introducing the harmonic approximation for the potential-energy surface. The latter approximation corresponds in fact to the reaction coordinate curve being a straight line in $n$-dimensional space and to the remaining coordinate curves lying in a hyperplane. Normal to this hyperplane is the reaction path in this massweighted configuration space, a path which is distinct from the reaction coordinate. They are cotangential at the saddle-point.

${ }_{18}$ For example, WBK expression, such as that used by $\mathbf{R}$. P. Bell, Proc. Roy. Soc. (London) A148, 241 (1935) or that in Ref. 20. 
The coordinate curves for separable systems are of two types, open and closed, the former extending to infinity. Examples of the closed type are circles and ellipses. Such a curve would provide a convenient coordinate only if most of the scattering occurred over a relatively small portion of the arc. Then, the error of replacing an actual open path by an arc of a closed one becomes minor. We consider an example later.

\section{PROBABILITY DISTRIBUTION}

Near any point sufficiently far from the saddlepoint region, along the reaction coordinate $q^{r}$ in the separable potential energy surface, the potential energy varies relatively little with distance and the component of the momentum along this coordinate is real and relatively large. Classical statistics may then be used for $q^{r}$ there but not necessarily for the other coordinates. The wavefunction of all degrees of freedom but $q^{r}$ is denoted by $\psi^{\prime}$ :

$$
\psi^{\prime}=\prod_{\mu \neq r} \psi_{\mu}\left(q^{\mu_{1}}, \cdots, q^{\mu_{h_{\mu}}}\right)
$$

For any given value of $q^{r}$ and of the conjugate momentum $p_{r}$ the state of the remaining degrees of freedom can be regarded as describable by a (discrete) quantum number $\lambda$ : In the case of an isolated gaseous activated complex molecule confined in a volume, even the translational state can be regarded as quantized. In the case of any activated complex in solution, a macroscopic subsystem can be regarded as the complex. It can be placed in a box and the $n-1$ degrees of freedom regarded as given rise to discrete eigenvalues, characterized by the quantum number $\lambda$, for purposes of the present discussion.

Inspection of the separated equations (9) and (A4) (Appendix I) shows that $\psi^{\prime}$ depends on the separation constants $\alpha_{1}, \cdots, \alpha_{m}$, and that for any given value of $q^{r}$ these constants determine $\lambda$ and $p_{r}$, and conversely. Unlike $p_{r}$ and $q^{r}$ the $\alpha$ 's are constants of the motion, however.

The probability of the system being in a quantum state described by $\lambda$ and of being in any small element $\Delta q^{r} \Delta p_{r}$ is denoted by $P\left(\lambda, q^{r}, p_{r}, T\right) \Delta q^{r} \Delta p_{r}$. We suppose that the reactants are in statistical equilibrium with this system. Since the probabilities of the system being in $\Delta q^{\tau} \Delta p_{r}$ and of having any given value of $\lambda$ are independent and since the number of quantum states in $\Delta q^{r} \Delta p_{r}$ is $\Delta q^{r} \Delta p_{r} / h$, one obtains

$$
P\left(\lambda, p_{r}, q^{r}, T\right)=\exp \left[-\alpha_{1}\left(\lambda, p_{r}\right) / k T\right] / h Q_{1},
$$

where the given values of $p_{r}, q^{r}$, and $\lambda$ automatically fix the total energy $\alpha_{1}$ and where $\exp \left(-\alpha_{1} / k T\right)$ is the Boltzmann factor. $Q_{1}$ is the partition function of the reactants.

In any one of these quantum states we may take $\Delta q^{r}$ so small that none of the quantities $\phi_{\mu \nu}, g^{i j}$ or $g$ vary over it. When a system is in any one of these quantum states its probability of being in any volume element

$$
g^{\frac{1}{2}} \prod_{i=1}^{n} d q^{i}
$$

can be written as

$$
\left|\psi^{\prime}\right|^{2} A\left(q^{r}\right) g^{\frac{1}{2}} \prod_{i=1}^{n} d q^{i}
$$

where $A\left(q^{r}\right)$ is a normalizing factor for this state. In this classical approximation for the $q^{r}$ coordinate, $A\left(q^{r}\right)$ can be treated as a constant over $\Delta q^{r}$. Integration over all $q^{i}$ shows that $A\left(q^{r}\right)$ equals

$$
\frac{1}{\Delta q^{r}} \int \mid \psi^{\prime} i g^{2} \prod_{i \ngtr r} d q^{i}
$$

Thus, the probability of find the system in the range

$$
d p_{r} \prod_{i=1}^{n} d q^{i}
$$

and in the state $\lambda$ is

$$
P_{(p)}\left(\lambda, p_{r}, \mathbf{q}\right) d p_{r} \prod_{i=1}^{n} d q^{i},
$$

where

$$
\begin{aligned}
& P_{(p)}\left(\lambda, p_{r}, \mathbf{q}\right) \\
& \quad=\exp \left[-\alpha_{1}\left(\lambda, p_{r}\right) / k T\right]\left|\psi^{\prime}\right|{ }^{2} g^{\ddagger} / h Q_{1} \int \mid \psi^{\prime}{ }^{2} g^{\natural} \prod_{i \neq \sigma_{r}} d^{i}
\end{aligned}
$$

and $\mathbf{q}$ denotes the totality of coordinates $\left(q^{1}, \cdots, q^{n}\right)$.

For each (discrete) value of $\lambda$, any of the $\alpha_{k}$ depends continuously on $p_{r}$, except as noted below. The probability of finding the system in

$$
d \alpha_{k} \prod_{i=1}^{n} d q^{i}
$$

and in the state $\lambda$ is obtained by replacing $d p_{\text {r }}$ above by $\left(\partial p_{r} / \partial \alpha_{k}\right)_{\lambda} d \alpha_{k}$. Upon denoting this probability by

$$
P\left(\alpha_{k}, \lambda, \mathbf{q}\right) d \alpha_{k} \prod_{i=1}^{n} d q^{i}
$$

and evaluating $\left(\partial p_{r} / \partial \alpha_{k}\right)_{\lambda}$ in Appendix I we find

$$
\begin{aligned}
& P\left(\alpha_{k}, \lambda, \mathbf{q}\right) \\
& \quad=\exp \left(-\alpha_{1} / k T\right)\left|\psi^{\prime}\right|{ }^{2} g^{\frac{1}{3}} / h Q_{1} p_{r} \int\left|\psi^{\prime}\right|^{2} \phi^{r k} g^{t} \prod_{i x^{\prime}} d q^{i} .
\end{aligned}
$$

With the aid of (17) it is possible to express the reaction rate in a form involving summation and integration 
over the constants of the motion. This step was not possible from (15) or (16).

The above transformation from the $\left(p_{r}, \lambda\right)$ space to the $\left(\alpha_{k}, \lambda\right)$ space is permissible only for those $\alpha_{k}$ 's for which the denominator does not vanish, i.e., for which $\phi^{r k} \neq 0$. The vanishing of $\phi^{r k}$ implies, as one can see from Eq. (A10) of Appendix I, that $\alpha_{k}$ does not depend on $p_{r}$ when $\lambda$ is held constant. An example is cited later.

It is convenient to introduce the notation

$$
\left(\psi^{\prime}, \psi^{\prime}\right)_{r k}=\hbar \int\left|\psi^{\prime}\right| \frac{\phi^{r k} g^{\frac{1}{2}}}{f_{r}} \prod_{i \not b r} d q^{i}
$$

From Eq. (13) one has

$$
\phi^{r k} g^{\frac{1}{2}} / f_{r}=\phi \phi^{r k} \prod_{\mu \not \prec r} f_{\mu} .
$$

Since $\phi \phi^{r k}$ is the cofactor of $\phi_{r k}$ in the determinant $\phi$ [cf. Eq. (10)], it does not contain the $r$ th row $\phi_{r}$ and so does not depend on $q^{r}$; neither does $\prod_{\mu \neq r} f_{\mu}$ and, so, neither does $\left(\psi^{\prime}, \psi^{\prime}\right)_{r k}$. If the wavefunction in the $n$ dimensional system were normalized so that $\left(\psi^{\prime}, \psi^{\prime}\right)_{r 1}$ equalled unity, the wavefunction would be normalized to unit incident probability current. ${ }^{13}$

\section{CALCULATION OF THE REACTION RATE}

When the potential energy is measured relative to the potential energy of the most stable configuration of the activated complex, $Q_{\mathbf{1}}$ denotes the partition function of the reactants measured relative to this energy zero. We let $Q$ denote the usual partition function of the reactants, i.e., measured relative to the potential energy of their most stable configuration. If $\Delta U$ is the potential energy of the most stable configuration of the activated complex minus that of the most stable one of the reactants then $Q_{1}$ equals $Q \exp (\Delta U / k T)$.

To obtain $k_{\text {rate }}$ from (17), one notes that (17) is to be multiplied by the velocity $\dot{q}^{r}$, by $\prod_{i \neq r} d q^{i}$ and by the transmission coefficient $\kappa\left(\alpha_{k}, \lambda\right)$, then summed over all $\lambda$ and integrated over all values of $\alpha_{k}$ and over all $q^{i}(i \neq r)$. Upon observing ${ }^{19}$ that $\dot{q}^{r}$ equals $\phi^{r 1} p_{r}$ the

${ }^{19}$ The kinetic energy in classical mechanics is

$$
\frac{1}{2} \sum_{i, j=1}^{n} g_{i j} \dot{q}^{i} \dot{q}^{j}
$$

so that the momentum conjugate to $q^{i}, p_{i}$, is

$$
\sum_{j=1}^{n} g_{i j} \dot{q}^{j}
$$

In the systems being considered $g^{i j}$ vanishes when $i$ and $j$ belong to different sets. Since the reaction coordinate is a one-dimensional separable set $g^{i r}$ vanishes when $i \neq r$. It then follows that $g_{i r}$ also vanishes for $i \neq r$ and $g_{r r}$ equals $1 / g^{r r}$. Hence, $p_{r}$ equals $\dot{q}_{r} / g^{r r}$. Eq. (11) then shows that $p_{r}=\dot{q}^{\tau} / \phi^{r 1}$ since $h_{r}=1$ and there is only one $g^{r^{r} j}$, namely $g^{r r}$. final expression for $k_{\text {rate }}$ is found to be

$$
\begin{aligned}
& k_{\text {rate }}=\frac{k T}{h} \frac{\exp (-\Delta U / k T)}{Q} \int_{\alpha_{k}} \sum_{\lambda} \kappa\left(\alpha_{k}, \lambda\right) \\
& \times \exp \left(-\frac{\alpha_{1}}{k T}\right) \frac{\left(\psi^{\prime}, \psi^{\prime}\right)_{r 1}}{\left(\psi^{\prime}, \psi^{\prime}\right)_{r k}} \frac{d \alpha_{k}}{k T}, \quad(k=1, \cdots, m)
\end{aligned}
$$

where $\alpha_{1}$ is a function of $\alpha_{k}$ and $\lambda$.

For any preassigned value of $\alpha_{k}$, the remaining $\alpha$ 's take on discrete values, reflected by the quantum numbers $\lambda$. Accordingly, Eq. (20) can be rewritten in a slightly more symmetrical form:

$$
\begin{aligned}
k_{\text {rate }}=\frac{k T}{h} \frac{\exp (-\Delta U / k T)}{Q} & \int_{\alpha_{k}} \sum_{\alpha_{j} ; ; \neq \neq k k} \kappa(\alpha) \\
& \times \exp \left(-\frac{\alpha_{1}}{k T}\right) \frac{\left(\psi^{\prime}, \psi^{\prime}\right)_{r 1}}{\left(\psi^{\prime}, \psi^{\prime}\right)_{r k}} \frac{d \alpha_{k}}{k T},
\end{aligned}
$$

where $\alpha$ denotes totality of $\alpha^{\prime}$ 's, $\left(\alpha_{1}, \cdots, \alpha_{m}\right)$ and where each $\psi_{\mu}$ is a function of $\left(q^{\mu_{1}}, \cdots, q^{\mu_{h_{\mu}}}, \alpha_{1}, \cdots, \alpha_{m}\right)$. When $\alpha_{k}$ is taken to be $\alpha_{1}$, one finds

$$
k_{\mathrm{rate}}=\frac{k T}{h} \frac{\exp (-\Delta U / k T)}{Q} \int_{\alpha_{1}} \sum_{\alpha_{i} ; ; \neq 1} \kappa(\alpha) \exp \left(-\frac{\alpha_{1}}{k T}\right) \frac{d \alpha_{1}}{k T} .
$$

The lower limit on $\alpha_{1}$ is $-\Delta U$, though in practice the exact value of the lower limit hardly affects $k_{\text {rate }}$, since most of the contribution to the integral comes from values of $\alpha_{1}$ near the top of the barrier, i.e., values near zero.

In calculations of the rate constant itself, Eq. (22) appears to be the most useful form of (21), at least when most of the activated complexes are formed in low vibrational states for coordinates interacting with the reaction coordinate: Its use avoids the calculation of $\left(\psi^{\prime}, \psi^{\prime}\right)_{r 1}$ and $\left(\psi^{\prime}, \psi^{\prime}\right)_{r k}$ and also permits a direct comparison to be made of the values of $k \exp \left(-\alpha_{1} / k T\right)$ for the curvilinear and for the usual Cartesian approximation. The comparison can be made as a function of $\alpha_{1}$ for each vibrational state of the activated complex. On the other hand, for an examination of the manner in which the curvilinear formula for $k_{\mathrm{rate}}$ approaches the Cartesian one, Eq. (21) with some $k$ other than 1 is useful, as shown in some examples described later.

\section{TRANSMISSION COEFFICIENT}

The transmission coefficient $k(\alpha)$ is the ratio of transmitted to incident probability currents for the given value of the constants of the motion, $\alpha$. To calculate $\kappa$ an expression for the probability current in curvilinear coordinates must be used. This expression has been given elsewhere in terms of the wavefunction along this coordinate. ${ }^{13}$ The phase integral 
method can be used for a semiclassical estimate of $\kappa$, incidentally, since the Schrödinger-type equation for the reaction coordinate $[\mathrm{Eq}$. (9) with $\mu=r]$ can readily be adapted to this calculation. An improved formula for the semiclassical estimate, one which takes cognizance of the proximity of the two transition points for the case of energies below the top of the barrier, is available and is discussed elsewhere..$^{20}$

\section{APPLICATION OF EQ. (21)}

By way of example, we consider a three-center reaction, $A+B C \rightarrow A B+C$, proceeding via a linear collision complex. In mass-weighted configuration space the reaction path is curved.9,21 As mentioned earlier, this path can be matched in the local approximation by the corresponding path for a surface which permits separation of variables. Some of the matching has been discussed previously ${ }^{7}$ and, in the following discussion, is regarded as having been performed.

The plane defined in mass-weighted configuration space by the tangent and principal normal to the reaction path, the osculating plane of this path, can be called the "plane of reaction." In many diagrams in the literature $\mathrm{e}^{9,21}$ one assumes for simplicity that the reaction coordinate lies in a plane determined only by the $\mathrm{AB}$ and $\mathrm{BC}$ interatomic distances, mass-weighted as in Eq. (3). The remaining degrees of freedom are taken to be dynamically uncoupled from these two in those discussions. We consider this special case first.

In terms of the properties of a suitable coordinate system for matching of the potential-energy functions, the latter assumption leads to a choice of a cylindrical coordinate system: The $Z$ axis, which is normal to the plane of reaction, represents the set of all degrees of freedom but the $\mathrm{AB}$ and $\mathrm{BC}$ distances. The latter two coordinates can be used to describe any point in the plane. In Eq. (6) one has then three sets, $m=3$. With proper choice of coordinates one can diagonalize the kinetic energy contribution of the coordinates in the plane of reaction and normalize their coefficients such that for two Cartesian coordinates in this plane the $g^{s s}$ are unity. One can choose a scaling factor for the remaining coordinates (represented metrically by the $Z$ axis) so that

$$
\underset{(\mu=0)}{\operatorname{det} g^{\mu_{i} \mu_{j}}}
$$

is unity.

When the reaction coordinate is arbitrarily assumed to be a straight line, as in the usual treatment in the literature, ${ }^{1,21}$ it suffices to consider only two sets of coordinates, as in Example 1 below, instead of three sets.

${ }^{20} \mathrm{R}$. A. Marcus (to be published). The discussion is based on some results contained in a recent book by J. Heading, $A \boldsymbol{n}$ Introduction to Phase-Integral Methods (Methuen \& Company, Ltd., London, 1961).

${ }^{21}$ For example, R. E. Weston, Jr., J. Chem. Phys. 31, 892 (1959).
It is shown there that this neglect of reaction coordinate curvature permits one to obtain the standard literature rate expression from Eq. (21). The effect of curvature is then discussed in Example 2 using a circular cylinder metric and in Examples 3 and 4, more briefly, using elliptic cylinder and parabolic cylinder metrics. When the reaction is symmetrical, e.g., when $\mathrm{A}+\mathrm{BA} \rightarrow \mathrm{AB}+$ $A$, the vibration of the activated complex in the plane of reaction is rectilinear and Examples 2 and 3 apply. However, when the vibrational coordinate curve in the plane of reaction is not a straight line, and when one wishes to describe this curvature, one of the coordinate systems in Examples 3 or 4 may be used. The various $\phi_{\mu \nu}$ 's, $f_{\mu}$ 's, and $g^{s t}$ 's are known for a variety of coordinate systems. ${ }^{15,16}$

Cylinder coordinate systems can also be used to represent a more general picture of the three-center reaction in which additional coordinates besides the two bond distances are permitted to contribute to the plane of reaction. If one considers the $n$-dimensional curve describing the reaction path in mass-weighted configuration space, the osculating plane of the path would be taken to be the plane normal to the $Z$ axis. One then proceeds as before.

Some applications of Eq. (22) to the calculation of the rate constant using some of the following coordinate systems and reaction coordinates will be reported elsewhere. ${ }^{10}$ The present discussion is concerned instead with an amplification of the preceding discussion, with showing in Example 1 how the usual activated complex theory equation in the literature is a special case of Eq. (21), and with discussing in Examples 2 and 3 the approach of the curvilinear formulas embodied in (21) to the Cartesian one. A suitable choice of $k$ for the latter purpose is described. Curvilinear coordinate systems other than those discussed in the following examples might be used instead, when one avoids the Stäckel-Robertson formalism.

\section{Example 1. Cartesian Metric}

With $m$ equal to 2 the $\phi_{\mu \nu}$ 's for the Cartesian metric are given by ${ }^{16}$

$$
\left(\begin{array}{ll}
\phi_{11} & \phi_{12} \\
\phi_{21} & \phi_{22}
\end{array}\right)=\left(\begin{array}{rr}
1 & -1 \\
0 & 1
\end{array}\right) .
$$

One must first determine which $\phi^{r k}$ 's in Eq. (21) vanish. Since $\phi \phi^{i j}$ is the cofactor of $\phi_{i j}$ in $\phi$, it is seen from (23) that only $\phi^{12}$ is zero. Thus, if $q^{1}$ is selected as the reaction coordinate, the continuous variable $\alpha_{k}$ in Eq. (21) should not be taken to be $\alpha_{2}$. Otherwise, it makes no difference whether $q^{1}$ or $q^{2}$ is selected. We choose $q^{2}$ to be $q^{r}$ and $\alpha_{k}$ to be $\alpha_{2}$. The $\psi_{2}$ equation for the reaction coordinate then depends only on $\alpha_{2}$, so $\kappa$ depends only on $\alpha_{2}$. The $\psi_{1}$ equation for the other 
degrees of freedom depends only on $\left(\alpha_{1}-\alpha_{2}\right)$, which thereby takes on discrete values. They are denoted below by $\alpha_{1}^{\prime}$. Since $\alpha_{1}$ equals $\left(\alpha_{1}-\alpha_{2}\right)+\alpha_{2}$, Eq. (21) then reduces to (24):

$$
k_{\text {rate }}=\frac{k T}{h} \frac{Q^{\ddagger}}{Q} e^{-\Delta U / k T} \int \kappa\left(\alpha_{2}\right) e^{-\alpha_{2} / k T} \frac{d \alpha_{2}}{k T},
$$

where $Q^{\ddagger}$ is the partition function of the activated complex, $\sum_{\alpha_{1^{\prime}}} \exp \left(-\alpha_{1}^{\prime} / k T\right)$. When tunneling is neglected, $k\left(\alpha_{2}\right)$ vanishes for $\alpha_{2}<0$. In the same classical approximation it is unity for $\alpha_{2} \geq 0$, as one sees at once from the $\psi_{2}$ equation.

Equation (24) is equivalent to the standard equation in the literature for the activated complex theory. ${ }^{1,21}$

\section{Example 2. Circular Cylindrical Coordinates}

We take $q^{1}=r$ and $q^{2}=\cos \theta$, when $r$ and $\theta$ are the usual polar coordinates. The $\phi_{\mu \nu}$ 's are given by ${ }^{16}$ :

$$
\left(\begin{array}{ccc}
\phi_{11} & \phi_{12} & \phi_{13} \\
\phi_{21} & \phi_{22} & \phi_{23} \\
\phi_{31} & \phi_{32} & \phi_{33}
\end{array}\right)=\left(\begin{array}{ccc}
1 & \left(-1 / q^{1}\right)^{2} & -1 \\
0 & 1 /\left(1-q^{2^{2}}\right) & 0 \\
0 & 0 & 1
\end{array}\right) \text {. }
$$

The reaction coordinate is taken to be along the arc of a circle and, therefore, to be $q^{2}$. Use of this coordinate system implies that the potential-energy surface, plotted in mass-weighted coordinates, is symmetrical along $q^{r}$ about the saddle-point region.

From (25) $\phi \phi^{21}, \phi \phi^{22}$, and $\phi \phi^{23}$ are found to be $1 / q^{1^{2}}, 1$, and 0 , respectively. Hence, $\alpha_{1}$ or $\alpha_{2}$, but not $\alpha_{3}$, may be picked as $\alpha_{k}$ to avoid a singular transformation. To show the approach of Eq. (21) to a Cartesian formula in this case we select $\alpha_{2}$, since the $\psi_{2}$ equation (and hence $\kappa$ ) depends only on $\alpha_{2}$. The $\psi_{3}$ equation depends only on $\alpha_{3}$, which takes on discrete values. The $\psi_{1}$ equation depends on $\alpha_{1}-\alpha_{3}-\alpha_{2} /\left(q^{1}\right)^{2}$. For any preassigned value of $\alpha_{2}, \alpha_{1}-\alpha_{3}$ then takes on discrete values. Let $\alpha^{\prime}=\alpha_{1}-\alpha_{3}$. Noting that $\alpha_{1}=\alpha_{3}+\alpha^{\prime}$, Eq. (21) becomes

$k_{\text {rate }}=\frac{k T}{h} e^{-\Delta U / k T} \frac{Q_{3}^{\ddagger}}{Q} \int_{\alpha_{2}} \kappa\left(\alpha_{2}\right)\left[\sum_{\alpha^{\prime}} e^{-\alpha^{\prime} / k T}\left\langle\frac{1}{{q^{1}}^{1^{2}}}\right\rangle_{\alpha^{\prime}, \alpha_{2}}\right] \frac{d \alpha_{2}}{k T}$,

where $Q_{3}^{\ddagger}$ denotes $\sum_{\alpha_{3}} \exp \left(-\alpha_{3} / k T\right)$, the partition function for all coordinates of the activated complex other than $q^{1}$ and $q^{2}$, and where \langle\rangle denotes an average

${ }^{22}$ A. B. Grieve, Analytical Geometry (G. Bell and Sons, Ltd., London, 1948), p.| 85.

${ }_{23}$ For example, O. Schreier and E. Sperner, Introduction to Modern Algebra and Matrix Theory (Chelsea Publishing Company, New York, 1959), 2nd ed., p. 101, 98. value:

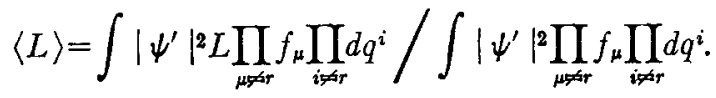

The average depends on the subscripts indicated in (26), $\alpha^{\prime}$ and $\alpha_{2}$, because $\psi^{\prime}$ depends on them.

The vibrational motion in the plane of reaction (coordinate $q^{1}$ ) is coupled to that along the reaction coordinate $q^{2}$ via a constant of the motion, $\alpha_{2}$. The remaining motions of the complex, described by the $\psi_{3}$ equation, are dynamically uncoupled from these two.

The resemblance of Eq. (26) to the Cartesian result (24) is increased by introducing $\alpha_{1}{ }^{\prime}, \alpha_{2}{ }^{\prime}$, and $\kappa^{\prime}$ :

$$
\begin{aligned}
\alpha_{1}{ }^{\prime} & =\alpha_{1}-\alpha_{3}-\alpha_{2} / q_{0}{ }^{{ }^{2}}, \\
\alpha_{2}{ }^{\prime} & =\alpha_{2} / q_{0}{ }^{{ }^{2}}, \\
\kappa^{\prime}\left(\alpha_{2}{ }^{\prime}\right) & \equiv \kappa\left(\alpha_{2}\right),
\end{aligned}
$$

where $q_{0}{ }^{1}$ is the value of $q^{1}$ at the saddle point. Equation (26) becomes

$$
\begin{aligned}
k_{\mathrm{rate}}=\frac{k T}{h} e^{-\Delta U / k T} \frac{Q_{3}{ }^{\ddagger}}{Q} \int_{\alpha_{2}{ }^{\prime}} & {\left[\sum_{\alpha_{1^{\prime}}} \exp \left(-\frac{\alpha_{1}{ }^{\prime}}{k T}\right)\left\langle\frac{q_{0}{ }^{{ }^{2}}}{{q^{2}}^{\mathbf{2}^{2}}}\right\rangle\right] \kappa^{\prime}\left(\alpha_{2}{ }^{\prime}\right) } \\
& \times \exp \left(-\alpha_{2}{ }^{\prime} / k T\right)\left(d \alpha_{2}{ }^{\prime} / k T\right) .
\end{aligned}
$$

The transition of (31) to (24) may be seen by studying the behavior of the former in the region where the curvature of each coordinate curve near the saddle point is small. The curvature of the $q^{1}$ coordinate curve is already zero. The curvature of the $q^{2}$ curve passing through the saddle point is equal to the reciprocal of the radius vector there, i.e., to the value of $1 / q^{1}$ for that curve. When $q^{1}$ is very large, $q^{1}$ undergoes only small fractional variations during any typical motion of the activated complex, and may be replaced in (31) by its value at the saddle point, $q_{0}{ }^{1}$. Similarly, in the $\psi_{1}$ equation, the $\alpha_{2} / q^{{ }^{2}}$ becomes $\alpha_{2} / q_{0}{ }^{2}$. Then, $\alpha_{1}{ }^{\prime}$ becomes an eigenvalue of the $\psi_{1}$ equation and, also, the sum in (31) becomes independent of $\alpha_{2}$. One obtains

$\underset{\mathrm{rate}}{\underset{\mathbf{q} 0^{1} \rightarrow \infty}{\longrightarrow}} \rightarrow \frac{k T}{h} \frac{Q^{\ddagger}}{Q} e^{-\Delta U / k T} \int_{\alpha_{2^{\prime}}} \kappa^{\prime}\left(\alpha_{2}^{\prime}\right) \exp \left(-\frac{\alpha_{2}^{\prime}}{k T}\right) \frac{d \alpha_{2}^{\prime}}{k T}$,

where $Q^{\ddagger}$ equals $Q_{3}^{\ddagger} \sum_{\alpha_{1^{\prime}}} \exp \left(-\alpha_{1}^{\prime} / k T\right)$. It is the partition function of the activated complex for all coordinates but the reaction coordinate. Equation (32) is in fact identical with (24).

In Appendix II it is also shown that the separated wave equations become those for the Cartesian case. This reduction and that given below for Example 3 can presumably also be used to suggest physically motivated approximations in (31) to simplify the integration-summation when the curvature consequences of the metric in the vicinity of the saddle point are slight. 
We conclude this section with some remarks on the use of Eq. (22) to calculate $k_{\text {rate. }}$. For this calculation it is convenient to introduce $\alpha_{1}{ }^{\prime}$ defined above (it equals $\alpha_{1}-\alpha_{3}$ ) and to note that $d \alpha_{1}$ can be replaced by $d \alpha_{1}{ }^{\prime}$. Equation (22) may then be summed over the discrete values of $\alpha_{3}$, yielding

$k_{\mathrm{rat \theta}}=\frac{k T}{h} e^{-\Delta U / k T} Q_{3^{*}} \int_{\alpha_{1^{\prime}}} \sum_{\alpha_{2}} \kappa\left(\alpha_{2}\right) \exp \left(-\frac{\alpha_{1}{ }^{\prime}}{k T}\right) \frac{d \alpha_{1}{ }^{\prime}}{k T}$.

Noting that for any given value of $\alpha_{1}^{\prime}$ the solution of the $\psi_{1}$ equation yields the allowed values of $\alpha_{2}, \kappa$ may be calculated for these $\alpha_{2}$ 's, summed as in (33), and finally the latter may be integrated. For systems in which almost all of the activated complexes are formed in their lowest $q^{1}$ vibrational states the first term in the sum suffices.

\section{Example 3. Elliptic Cylinder Coordinates}

This coordinate system contains an added parameter $d$ and therefore offers a somewhat more flexible choice in matching the separable and nonseparable energy surfaces over a larger distance from the saddlepoint. When $2 d$, the distance between the foci of the confocal ellipses vanishes, the coordinate curves degenerate into those for the circular cylinder system and (34) and (35) become identical with (25) and (26). Coordinates $q^{1}$ and $q^{2}$ are introduced. They equal $\left(r_{1}+r_{2}\right) / 2$ and $\left(r_{1}-r_{2}\right) / 2 d$, where $r_{1}$ and $r_{2}$ are the distances of a point in the plane to the two foci.

The matrix of $\phi_{\mu \nu}$ 's is ${ }^{16}$

$$
\left(\begin{array}{ccc}
1 & -1 /\left(q^{1^{2}}-d^{2}\right) & -1 \\
d^{2} & 1 /\left(1-q^{2^{2}}\right) & -d^{2} \\
0 & 0 & 1
\end{array}\right)
$$

As a reaction coordinate one may select a hyperbola or the arc of ellipse; $q^{r}$ then becomes $q^{1}$ and $q^{2}$, respectively. We consider the $r=2$ case first.

\section{a. Reaction Coordinate Along Arc of Ellipse}

When the potential-energy surface, plotted in massweighted coordinates, is symmetrical about the activated complex region, the saddle point will occur on the major or minor axis of the ellipse, i.e., at $q^{2}= \pm 1$ or 0 , respectively. The quantities $\phi \phi^{21}, \phi \phi^{22}, \phi \phi^{23}$ equal $\left(q^{1^{2}}-d^{2}\right)^{-1}, 1$, and 0 , respectively. Accordingly, only the choice of $\alpha_{k}=\alpha_{3}$ is forbidden in Eq. (21). We take $\alpha_{k}=\alpha_{2}$ to show the approach of (21) to the Cartesian formula.

The $\psi_{3}$ equation depends only on $\alpha_{3}$, which therefore has discrete values. In the $\psi_{1}$ equation, $\sum \alpha_{\nu} \phi_{1}$ equals $\alpha_{1}-\alpha_{3}-\alpha_{2}\left(q^{1^{2}}-d^{2}\right)^{-1}$. For a preassigned $\alpha_{2}, \alpha_{1}-\alpha_{3}$ therefore has discrete values. The $\psi_{2}$ equation is to be solved to obtain $\kappa$. It contains $\sum_{\nu} \alpha_{\nu} \phi_{2 v}$, i.e., $\left(\alpha_{1}-\alpha_{3}\right) d^{2}+\alpha_{2}\left(1-q^{2^{2}}\right)^{-1} . \kappa$ is seen to depend on $\alpha_{1}-\alpha_{3}$ and on $\alpha_{2}$.

Introducing $\alpha^{\prime}=\alpha_{1}-\alpha_{3}$ the expression for $k_{\text {rate }}$ becomes

$$
\begin{aligned}
k_{\text {rate }}=\frac{k T}{h} e^{-\Delta U / k T} \frac{Q_{3}}{Q} \int_{\alpha_{2}} & \sum_{\alpha_{1}} k\left(\alpha_{2}, \alpha^{\prime}\right) \\
& \times e^{-\alpha^{\prime} / k T}\left\langle\frac{1}{{q^{12}-d^{2}}^{2}}\right\rangle \frac{d \alpha_{2}}{k T},
\end{aligned}
$$

where $Q_{3}{ }^{\ddagger}=\sum \alpha_{\alpha_{3}} \exp \left(-\alpha_{3} / k T\right)$ and where the average, \langle\rangle , is defined in Eq. (27).

The approach of expression (35) to (24) can be seen by examining the behavior or (35) when the curvilinear effects of the metric are small near the saddle point.

\section{b. Reaction Coordinate along a Hyperbola}

For a symmetrical potential energy surface the saddle point occurs on the transverse axis ${ }^{22}$ of the hyperbola, i.e., at $q^{1}=d$. Since $\phi \phi^{11}, \phi \phi^{12}$, and $\phi \phi^{13}$ equal $\left(1-q^{2^{2}}\right)^{-1},-d^{2}$ and 0 , respectively, only the choice of $\alpha_{k}=\alpha_{3}$ is forbidden in (21). When the approach to the Cartesian case is investigated it is noted in Appendix II that $d$ tends to zero. Thus, only the choice of $\alpha_{k}=\alpha_{1}$ is permissible for investigating the approach. For calculation of $k_{\text {rate }}$ in general, however, $\alpha_{k}=\alpha_{1}$ or $\alpha_{2}$ can be used. For brevity, a detailed investigation of the approach is omitted.

\section{Example 4. Parabolic Cylinder Coordinates}

Because the symmetry of the two parabolic coordinates, it makes no difference which of the two is selected as reaction coordinate. If the potential-energy surface is symmetrical about the activated complex region, the saddle point occurs on the axis of the confocal parabolas.

\section{EFFECT OF ROTATIONAL MOTION ON $\boldsymbol{k}_{\text {rate }}$}

In calculations of reaction rate constants rotationvibration interactions are normally neglected. They were omitted in the derivation of (21) and (22) by making some of the $g^{\text {rs }}$ constant and, thereby, some of the $\phi_{\mu \nu}$ constant or zero. The influence of the interactions will now be considered for completeness. Although this neglect is normally quite justified, there are a number of related problems where their inclusion is a matter of some importance. For example, the rates of unimolecular dissociation are influenced by the centrifugal potential in the molecule. Calculations of the dependence of the dissociation rate on the energy of a decomposing molecule should allow for it.

In the first approximation a "diatomic approximation" may suffice. In the latter only the rotationalvibrational interaction associated with a rotation involving the two largest moments of inertia is considered and one may proceed as follows. 
In this "diatomic approximation," the Schrödinger equation has a form permitting separation of variables ${ }^{13}$ according to the formalism described earlier. The wave equation is first separated into equations for the translational (center of mass), rotational and internal motions using this formalism. The equation for internal motion now contains a rotational constant of the motion, which appears in a centrifugal potential term. The sum of the potential energy and of this centrifugal potential has a saddle point when the original potential energy function has one and when the centrifugal distortion is not too large. A suitable system of internal coordinates is then introduced to permit this effective potential energy surface to be approximated by a surface permitting separation of variables, as described previously. Thereby, the choice of the reaction coordinate now depends on the rotational state of the activated complex. With this qualification in mind, Eqs. (21) and (22) again apply, but now the summation over the rotational $\alpha$ should be made only after the other summations and the integration have been performed.

A slightly more general approach would be to neglect the vibrational angular momentum as before and to treat the activated complex as a symmetric top. Although separation of rotational from internal motion does not fall within the previously described formalism, one can easily effect the separation in a standard way. The separated equation for the internal motion contains rotational constants of the motion and can be treated as in the preceding paragraph. Once again, the summation over the rotational $\alpha$ 's in Equations (21) and (22) would be performed last.

\section{APPENDIX I. EVALUATION OF $\left(\partial p_{r} / \partial \alpha_{k}\right)_{\lambda}$}

We first note that when a set $\mu$ contains a single coordinate $q^{r}$ Eq. (9) reduces to:

$$
-\frac{\hbar^{2}}{2 f_{r}} \frac{\partial}{\partial q^{r}} f_{r} \frac{\partial \psi_{r}}{\partial q_{r}}+X_{r} \psi_{r}=\sum_{\nu=1}^{m} \alpha_{\nu} \phi_{r \nu} \psi_{r}
$$

The semiclassical approximation for $\psi_{r}$ is then derived in the standard way by letting $\psi$ equal $\exp \left(i S^{r} / \hbar\right)$, with $S^{\tau}$ expanded in a power series in $\hbar$ :

$$
S^{r}=S_{0}{ }^{r}+(\hbar / i) S_{1}{ }^{r}+(\hbar / i)^{2} S_{2}{ }^{r}+\cdots .
$$

Retention of only the leading term yields the classical expression

$$
\frac{1}{2}\left(\partial S_{0} / \partial q^{\tau}\right)^{2}+X_{r}=\sum_{\nu=1}^{m} \alpha_{\nu} \phi_{r \nu}
$$

$S_{0}$ is a function of $q^{r}$ and of the $\alpha^{\prime}$ s, and $\partial S_{0} / \partial q^{r}$ is the momentum $p_{r}$ conjugate to $q^{r}$. Hence, we have

$$
\frac{1}{2} p_{r}{ }^{2}+X_{r}\left(q^{r}\right)=\sum_{\nu=1}^{m} \alpha_{\nu} \phi_{r \nu}\left(q^{r}\right) .
$$

We also need (A5), obtained by multiplying (9) by $\psi_{\mu}{ }^{*}$ and integrating over $d \mathbf{q}^{\mu}$, a symbol for the product

$$
\begin{gathered}
\prod_{i=1}^{h_{\mu}} d q^{\mu_{i}} \\
\int \psi_{\mu}{ }^{*}\left(H_{\mu}+X_{\mu} f_{\mu}\right) \psi_{\mu} d \mathbf{q}^{\mu}=\sum_{\nu=1}^{m} \alpha_{\nu} \int \Phi_{\mu \nu} f_{\mu} \psi_{\mu}{ }^{*} \psi_{\mu} d \mathbf{q}^{\mu}
\end{gathered}
$$

Continuous variation of $p_{r}$ at constant discrete quantum number $\lambda$ will cause a continuous variation in some of the $\alpha_{\nu}$ 's, and zero variation in the others. Since the $\alpha_{\nu}$ 's enter the separated equations, some of the $\psi_{\mu}$ 's are continuously altered, with no change in number of nodes, while others are unaffected. On letting the $\alpha_{\nu}$ and $\psi_{\mu}$ in each of these $m-1$ equations (A5) (with $\mu \neq r$ ) undergo their variations resulting from a change in $p_{r}, \delta p_{r}$, and noting that the differential operator is Hermitian, ${ }^{7}$ one finds

$$
\begin{gathered}
\int \delta \psi_{\mu}{ }^{*}\left(H_{\mu}+X_{\mu} f_{\mu}-\sum \alpha_{\nu} \phi_{\mu \nu} f_{\mu}\right) \psi_{\mu} d \mathbf{q}^{\mu} \\
+\int \delta \psi_{\mu}\left(H_{\mu}+X_{\mu} f_{\mu}-\sum \alpha_{\nu} \phi_{\mu \nu} f_{\mu}\right) \psi_{\mu}{ }^{*} d \mathbf{q}^{\mu} \\
=\sum_{\nu=1}^{m} \delta \alpha_{\nu} \int \phi_{\mu \nu} f_{\mu} \psi_{\mu}{ }^{*} \psi_{\mu} d \mathbf{q}^{\mu} .
\end{gathered}
$$

Because of Eq. (9), the left-hand side vanishes for the exact wavefunction. Hence,

$$
\sum_{v=1}^{m} \delta \alpha_{\nu} \int \phi_{\mu \nu} f_{\mu} \psi_{\mu}{ }^{*} \psi_{\mu} d \mathbf{q}^{\mu}=0 \quad \mu \neq r
$$

From Eq. (A4) one also obtains:

$$
\sum_{\nu=1}^{m} \delta \alpha_{\nu} \phi_{r v}=p_{r} \delta p_{r}
$$

On solving Eqs. (A6) and (A7) for $\delta \alpha_{k}$ in terms of $\delta p_{r}$ by means of Cramer's rule ${ }^{23}$ one finds

$$
\delta \alpha_{k}=\frac{p_{r} \delta p_{r}\left\{\sum_{P, p_{\mu} \neq k}(-1)^{P}\left[\prod_{\mu \neq ⿱} \int \phi_{\mu \nu_{\mu}} f_{\mu}\left|\psi_{\mu}\right|^{2} d \mathbf{q}^{\mu}\right]\right\}(-1)^{r+k}}{\sum_{P}(-1)^{P}\left[\prod_{\mu \neq t} \int \phi_{\mu v_{\mu}} f_{\mu}\left|\psi_{\mu}\right|^{2} d \mathbf{q}^{\mu}\right] \phi_{r_{r}}}
$$

where $P$ represents an even or odd permutation of the $\nu_{\mu}(i=1$ to $m)$ from the standard order $1, \cdots, m$. By interchanging the order of operations in the denominator of (A8), it can also be written as

$$
\int \sum_{P}(-1)^{P} \phi_{r v_{r}} \prod_{\mu \neq r}\left(\phi_{\mu \nu_{\mu}} f_{\mu}\left|\psi_{\mu}\right|^{2} d \mathbf{q}^{\mu}\right) .
$$


If $\phi$ denotes the determinant of the $\phi_{\mu \nu}$, this integral becomes

$$
\int \phi \prod_{\mu \not \nexists r} f_{\mu}\left|\psi_{\mu}\right|^{2} d \mathbf{q}^{\mu}
$$

Similarly, the numerator of (A8) becomes $p_{r} \delta p_{r}$ multiplied by such an integral with $\phi$ replaced by an $m-1 \times m-1$ cofactor, namely, the cofactor of $\phi_{r k}$ in $\phi$. Because of (10) this cofactor equals $\phi \phi^{r k}$. One thus finds, with the aid of (18),

$$
\delta \alpha_{k}=p_{r} \delta p_{r}\left(\psi^{\prime}, \psi^{\prime}\right)_{r k} / \int\left|\psi^{\prime}\right|^{2} \phi \prod_{\mu \neq r r} f_{\mu} \prod_{i \neq r} d q^{i} .
$$

We may thus conclude that $\left(\partial p_{r} / \partial \alpha_{k}\right)_{\lambda}$ equals the value used in the text.

\section{APPENDIX II. APPROACH TO THE CARTESIAN CASE}

The curvature of a $q^{i}$-coordinate curve in the plane of reaction, $\kappa_{(i)}$, for any of the cylinder coordinate systems in Examples 1 to 4 equals $\mid\left(\partial \log g_{i i} / \partial q^{j}\right) / 2 g_{j j^{3}}$, where $q^{j}$ is the other coordinate in the plane of reaction. ${ }^{24}$ Since the element of arc length along the $q^{j}$-coordinate curve is $d s_{j}$, where

$$
d s_{j}=g_{j j^{\frac{1}{2}}} d q^{j},
$$

one can also write:

$$
\kappa_{(i)}=\frac{1}{2}\left|\left(\partial \log / \partial s_{j}\right) g_{i i}\right| .
$$

The $\psi_{1}$ equation, Eq. (A1) with $r$ replaced by $i$, can be converted to a form more suited to the present proof. We first write $g^{\frac{1}{2}}$ as $\left(g_{i i} g_{j j} g_{z}\right)^{\frac{1}{2}}$, where $g_{z}$ is the determinant of the $g_{s t}$ 's in the set $\mu=3$. In the case of no dynamic coupling between the set $\mu=3$ on the one hand, and the other two coordinates $q^{i}$ and $q^{j}$ on the other, $g_{z}$ is independent of $q^{i}$ and $q^{j}$. In the numerator

\footnotetext{
${ }^{24}$ This expression is deduced from the equation for the curvature in generalized coordinates. See, for example, Eqs. (16), (18), and (19b) of Ref. 7, with $N$ and $i$ replaced now by $i$ and $j$, respectively. The cited value of $\kappa_{(i)}$ follows at once from these equations, when one notes that all components of the curvature vector vanish except those in the plane of reaction.
}

of (A1) $f_{i}$ is now multiplied by

$$
\left(g^{\frac{1}{2}} \prod_{\mu \neq i} f_{\mu}\right)\left(g^{\frac{1}{2}} \prod_{\mu \neq i} f_{\mu}\right)^{-1} .
$$

Equations (13) and (A11) are next used. One then notes from (11) that $g^{i i}$ and $g^{j j}$ equal $\phi^{i 1}$ and $\phi^{j 1}$, since $i$ and $j$ are one-dimensional sets. Since $g_{z}, \prod_{\mu \neq i} f_{\mu}$, and $\phi \phi^{i 1}$ commute with $d / d q^{i}$ one finally obtains

$$
\begin{array}{r}
\left(d^{2} \psi_{i} / d s_{i}{ }^{2}\right)+\kappa_{(j)}{ }^{\prime}\left(d \psi_{i} / d s_{i}\right)-\left(2 / \hbar^{2}\right) \phi^{i 1}\left(X_{i}-\sum_{\nu=1}^{3} \alpha_{\nu} \phi_{i \nu}\right) \psi_{i} \\
=0, \quad(\mathrm{~A} 13
\end{array}
$$

where $\kappa_{(j)}^{\prime}$ equals $\frac{1}{2} \partial \log g_{i i} / \partial s_{j}$, and so has the same magnitude as $\kappa_{(j)}$. An equation similar to (A13) obtains for $\psi_{j}$, with $i$ and $j$ merely interchanged.

For the curvilinear case to approach the Cartesian one several conditions must be fulfilled. (a) The curvatures $\kappa_{(i)}$ and $\kappa_{(j)}$ must become negligible in (A13). (b) $\phi^{i 1} X_{i}$ in (A13) must tend to become a function of $q^{i}$ alone. (c) $\sum_{\nu} \phi^{i 1} \phi_{i \nu} \alpha_{\nu}$ in (A13) must become approximately constant over the relevant region of configuration space near the saddle point.

We consider Examples 2 and 3 individually.

i. Example 2. From (A11), (A12), and the known ${ }^{16}$ $g_{i i}$ 's the limiting case of vanishing curvatures of the curves passing through a point occurs when $q^{1}$ tends to infinity. The term $\phi^{i 1} \sum_{\nu} \alpha_{\nu} \phi_{i \nu}$ is given by (28) and (29) for $i=1$ and 2, respectively, with $q_{0}^{1}$ replaced by $q^{1}$. When $q^{1}$ has some large value, $q_{0}{ }^{1}$, this term becomes essentially a constant and so condition (c) above is fulfilled. Condition (b) is also fulfilled. Finally, the $\alpha_{2}{ }^{\prime}$ in (29) is the same as the one in (32) and the proof is complete.

ii. Example 3. From Eqs. (A11), (A12), and the known $g_{i i}$ 's one may find the conditions under which Conditions (a) to (c) above are fulfilled. They correspond to large $q^{1}$ and relatively small $d$. This case then reduces to the circular cylinder case, namely, Example 2 , and is not considered further. If the reaction coordinate is that in Example $3 \mathrm{~b}$, the choice $\alpha_{k}=\alpha_{2}$ is forbidden when $d$ tends to zero. Thus, in that case $\alpha_{k}$ must be chosen as $\alpha_{1}$ if one wishes to investigate the approach to the Cartesian case. 